\title{
UTOPOLIS - Die Stadt ohne Verkehrssystem als Chance für politische Bildung im GW-Unterricht. Ein Unterrichtskonzept für die Sekundarstufe I
}

\author{
*a0509723@unet.univie.ac.at, Institut für Geographie und Regionalforschung, Universität Wien \\ ***alexander.hoffelner@univie.ac.at, Institut für Geographie und Regionalforschung, Universität Wien \\ ***k ko.e@gmx.at, Institut für Geographie und Regionalforschung, Universität Wien \\ a0625924@unet.univie.ac.at, Institut für Geographie und Regionalforschung, Universität Wien
}

eingereicht am 17.06.2016; akzeptiert am 27.09.2016

\begin{abstract}
Dieser Artikel beschreibt ein handlungsorientiertes Unterrichtskonzept zum Thema „Verkehr“ für die Sekundarstufe I. Im Zentrum dieser Planung stehen der diskursive Prozess der Verkehrsplanung sowie die abschließende Planung eines Verkehrssystems der Stadt Utopolis aus der Sicht einer bestimmten Rolle. Das ständige Arbeiten in Kleingruppen soll die Entwicklung der einzelnen Schüler/innen bei diesem Thema umso mehr fördern, da individuelle Arbeitsweisen gefragt sind.
\end{abstract}

Keywords: Politische Bildung, Verkehr und Mobilität, Stadtplanung, Handlungskompetenz

\section{UTOPOLIS - The city without a transport system as an opportunity for political education in geo- graphy teaching}

This paper presents an activity-oriented teaching concept for secondary education on the topic of transport. The focus is on the discursive process in transport planning situations. A transport system for the city of Utopolis is to be developed from the point of view of a particular role. Ongoing work in small groups should help pupils to develop their argument on this topic.

Keywords: civic education, transport and mobility, urban planning, action competence

\section{$1 \quad$ Einleitung}

Utopolis ist eine fiktive Stadt, die etwa 20000 Menschen beheimatet. Gelegen an einem breiten Fluss, verfügt sie über einen historischen Stadtkern, Wohngebiete in- und außerhalb des Zentrums, ein Einkaufszentrum am Stadtrand, einen Campingplatz, ein Krankenhaus, Wälder und Seen im näheren Umfeld, ein Sportzentrum mit Stadion und sogar über einen kleinen Flughafen. Was jedoch fehlt, ist ein Verkehrssystem. Utopolis hat keine Straßen, Radwege oder öffentlichen Verkehrsmittel. Die Stadtplaner/innen haben schlichtweg darauf vergessen.

Aus dieser Situation heraus entsteht eine Chance für politische Bildung im Unterricht. Die Schüler/innen sind selbst tätig, planen ein neues Verkehrsnetz für die Stadt und trainieren und verbessern dabei ihre politische Handlungsfähigkeit. Wie das genau gehen soll und welche didaktischen und fachlichen Überlegungen dahinterstehen, zeigt der folgende Beitrag. ${ }^{1}$

\section{Didaktische Überlegungen}

Das Themengebiet „Verkehr" findet sich im Lehrplan der AHS für das Fach Geographie- und Wirtschaftskunde $(\mathrm{GW})$ in der Sekundarstufe I in der zweiten

\footnotetext{
Dieser Artikel ist das Resultat eines Seminars zur Unterrichtsplanung in Geographie und Wirtschaftskunde am Institut für Geographie und Regionalforschung der Universität Wien unter der Leitung von Herbert Pichler und Karin Dobler und der Erprobung in der Unterrichtspraxis am BG/BRG Eisenstadt unter der Betreuung von Susanne Reimann. Ihnen sei an dieser Stelle für ihr Feedback und ihre Unterstützung gedankt.
} 
Klasse. Dort sollen sich Schüler/innen mit der Tatsache auseinandersetzen, dass verschiedene Regionen bzw. Gebiete mit Verkehrsmitteln und -möglichkeiten unterschiedlich ausgestattet sind und insofern verschieden belastet werden (BMBF 2000, 4). Diese Tatsache soll unter dem Gesichtspunkt der politischen Bildung didaktisch aufgearbeitet werden. Das ergibt sich daraus, dass zum einen der Grundsatzerlass der politischen Bildung fordert, dass diese in allen Fächern - nach den bestehenden Möglichkeiten und Rahmenbedingungen - verwirklicht werden soll (BMBF 2015, 1). Zum anderen gibt das Fach Geographie- und Wirtschaftskunde per se eine politische Dimension vor, da es sich mit den Handlungen des Menschen im Raum auseinandersetzt und sich mit aktuellen und zukünftigen politischen Problemen zu beschäftigen hat (BMBF 2000, 1), wodurch verschiedene Politikfelder zu maßgebenden Elementen des GW-Unterrichts werden (Fassmann 2006).

Das Planen eines Verkehrssystems in verschiedenen Rollen und nach unterschiedlichen Interessen soll zur Förderung politischer Urteils- und Handlungskompetenz beitragen. Die politische Urteilskompetenz soll darin resultieren, dass Schüler/innen befähigt sind, politische Entscheidungen, Probleme und Kontroversen möglichst sach- und wertorientiert zu beurteilen, während die Handlungskompetenz die Fähigkeit, Fertigkeit und Bereitschaft beinhaltet, eigene Standpunkte $\mathrm{zu}$ artikulieren, andere Positionen nachzuvollziehen und aufzugreifen sowie an der Lösung von Problemen politischer und ökonomischer Art zu arbeiten (Krammer 2008, 9 f.). Gerade der zweite Aspekt erscheint in diesem Zusammenhang besonders wichtig, weil die politische Bildung im Sinne einer dialogisch-kommunikativen Bildung verstanden werden soll (Sutor 1984). Darin geht es um die Offenlegung des Politischen in der Kommunikation (Goll 2012, 106), was vor allem im letzten und der Ansicht der Autorinnen und Autoren nach wichtigsten Teil, der Reflexion über die Planung und die Kommunikation geschehen soll. Die Handlungsfähigkeit in der politischen Sphäre ist ein wesentlicher Aspekt politischer Freiheit. Um nämlich die eigene Freiheit realisieren zu können, muss "erlernt" werden, wie man am politischen Diskurs teilnimmt, wie eigene Ansichten und Standpunkte angemessen dargebracht werden können und wie in der Öffentlichkeit kompetent und erfolgreich gehandelt werden kann (Sander 2008, 91).

Fachlich gesehen geht es um die Erkenntnis, dass Raumordnung, Stadtplanung und Verkehrsentwicklung ein Produkt politischer Prozesse sind und damit im Spannungsfeld unterschiedlicher Interessen vollzogen werden. Das wird dadurch erschwert, dass es eine Vielzahl an Lebensstilen und -konzepten gibt, die allgemein zu einer Pluralisierung der Gesellschaft geführt haben. Das hat es der Raumplanung in den letzten Jahrzehnten nicht gerade einfacher gemacht (Langhagen-Rohrbach 2010, 16).

Die Methode, an welche die Unterrichtseinheit angelehnt ist, ist das Planspiel. Planspiele gehen von der Simulation eines Konfliktes oder Problems aus und sollen durch die Übernahme von Rollen und gleichzeitige Identifizierung mit diesen die Möglichkeit geben, politische bzw. ökonomische Entscheidungen zu treffen, ohne dafür reale Konsequenzen in Kauf nehmen zu müssen. Der Fokus liegt dabei auf strategischen Entscheidungen, wobei das Planspiel selbst im Rahmen vorgegebener Spielregeln abläuft. Planspiele sind recht aufwendig herzustellen und erfordern auch einen gewissen zeitlichen Rahmen, der oftmals außerhalb der Regelunterrichtszeit abzulaufen hat. Andererseits sind sie nahe an der Realität, weil sie ergebnisoffen sind (wie auch die Politik) und Flexibilität, Kreativität und Offenheit fordern und auch fördern. Damit können sich die Lernenden kritisch-konstruktiv mit den Lehrinhalten auseinandersetzen. Zentrale Ziele und Aspekte von Planspielen sind neben der bereits erwähnten, auch Selbstständigkeit, Verantwortungsbereitschaft, Teamfähigkeit, Kommunikationsfähigkeit und das Erlernen bestimmter (politischer) Methoden (Wiepcke 2011, 63 ff.).

Lernziele im Zuge dieser Unterrichtseinheit könnten folgendermaßen formuliert werden:

- Ich kann Probleme im öffentlichen Verkehr erkennen und beschreiben.

- Ich kann mögliche Lösungsansätze für diese Probleme finden.

- Ich kann mit anderen einen „Verkehrsplan“ erstellen, der verschiedene Interessen berücksichtigt.

- Ich kann meine eigene Funktion und mein Vorgehen im Prozess der Planung reflektieren.

Das Konzept spricht insbesondere das didaktische Prinzip der Handlungsorientierung an. Diese orientiert sich an den Interessen der Schüler/innen, hat ein vernünftiges Produkt als Ergebnis (keine reine „Beschäftigungstherapie") und verknüpft die Aspekte der Kopf- und Handarbeit (Jank \& Meyer 2011, 316 ff.). In diesem Zusammenhang spricht Wolfang Sitte von operativem Unterricht, also einem Unterricht, in dem durch die „tätige Auseinandersetzung der Schülerinnen und Schüler mit einem Lerngegenstand [...] ein durch geistiges und manuelles Tun zustandegekommenes sichtbares Produkt entsteht." (Sitte 2001, 305)

Beim handlungsorientierten Unterricht dürfen aber nicht alleine die Erlebnisse und Erfahrungen zählen, sondern diese müssen in weiterer Folge auf eine abstrakte, reflektierende Ebene gehoben werden. Handlungen sind unmittelbar mit dem Denken verbunden, durch das sie strukturiert werden müssen. 
(Hellmuth \& Klepp 2010, 143 f.) Auch in diesem Sinne ist die anschließende Reflexion ein zentraler Punkt der Sequenz, der auf keinen Fall vernachlässigt werden sollte.

Die Handlungsorientierung wird auch dadurch gefördert, dass das Konzept problemorientiert vorgeht, also sich mit dem Unterschied zwischen dem problematischen Ist-Zustand und dem erwünschten SollZustand beschäftigt, also die Brücke zwischen der Stadt ohne Verkehrsnetz und der Stadt, die ein Verkehrsnetz besitzt, das möglichst viele Interessen berücksichtigt, schlägt (Hellmuth \& Klepp 2010, 144).

\section{Ablauf der Unterrichtseinheit}

Am Beginn stellt die Lehrperson den Schülerinnen und Schülern die Stadt Utopolis vor und konfrontiert sie mit dem Problem, dass diese Stadt über kein Verkehrssystem welcher Art auch immer verfügt. Die einzige Vorgabe in dieser Hinsicht ist der bereits existente Flughafen, der vor allem für den Tourismus eine wichtige Rolle spielt. Außerdem bekommen die Schüler/ innen nach dem Zufallsprinzip Rollen zugeteilt, welche sie im Rahmen des gesamten Spiels vertreten. Es handelt sich dabei um die Rollen „Schüler/in“, „Radfahrer/in“, „Tourist/in“ und „Lieferant/in“. Auf den Rollenkärtchen finden sich auch die Positionen und Forderungen, welche die Schüler/innen im Rahmen des Planspiels vertreten.

Vorerst werden die Lernenden in Gruppen zu zwei Personen derselben Rolle zugeteilt. Jede Zweiergruppe kann nun mit dem Plan erste Ideen entwickeln, wie sie das Verkehrsnetz für die Stadt anlegen würde. Diese Ideen werden im nächsten Schritt in den nach den Rollen eingeteilten Fachgruppen konkretisiert. Es gibt also dann die Fachgruppe „Radfahrer/innen“, die Fachgruppe „Touristinnen und Touristen“, usw. Diese Gruppen sollen gemeinsam wichtige Punkte erarbeiten und auch schriftlich festhalten, welche sie später in den gemischten Planungsgruppen durchsetzen wollen.

Anschließend gehen die Schüler/innen in die Planungsgruppen, die aus jeweils vier Personen bestehen, wobei jeweils eine Person einer der Rollen zugehört. In sämtlichen Gruppen sitzen eine Radfahrerin bzw. ein Radfahrer, eine Touristin bzw. ein Tourist usw. Nun gilt es, die eigenen Interessen, die vorhin in den Gruppen festgelegt wurden, in der Planung des Verkehrsnetzes möglichst erfolgreich zu vertreten. Dies wird dadurch erschwert und gleichzeitig realitätsnäher gemacht, indem die Stadt über ein beschränktes Budget verfügt. Dieses ist mit 80 Geldeinheiten (GE) festgelegt, wobei die verkehrstechnischen Einheiten jeweils bestimmte Preise haben (siehe Liste unten). Die Lehrpersonen stehen in diesem Arbeitsschritt

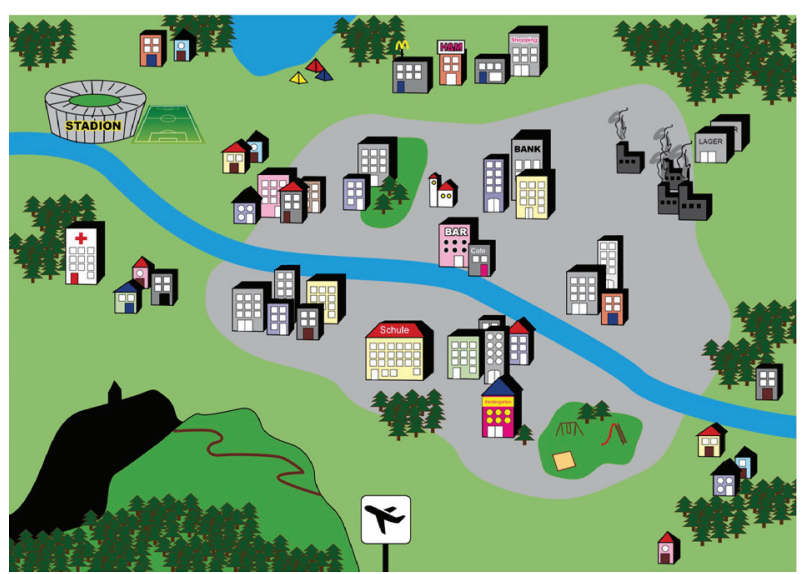

Abb. 1: Stadtplan von Utopolis ohne Infrastruktur (eigene Darstellung)

einerseits bei Fragen zur Verfügung, andererseits verwalten sie jene Materialien, die für die Planung auf den ausgedruckten Stadtplänen aufgelegt bzw. aufgeklebt werden können. Es gibt also eine Station im Klassenraum, wo mit dem zur Verfügung gestellten Budget, die Einheiten des Verkehrssystems gekauft werden können. Wenn der Plan auf A3 ausgedruckt wird, bietet es sich an im Bereich von $15 \mathrm{~cm}$ zu arbeiten. Eine Einheit Radweg wird also als Schnur in einer bestimmten Farbe in der Länge von $15 \mathrm{~cm}$ für $1 \mathrm{GE}$ an die Schüler/innen verkauft. Als Geld kann recht einfach der Inhalt eines Pokerkoffers verwendet werden. Elemente, welche über eine bestimmte Länge verfügen, können als Schnüre ausgegeben werden, punktuelle Elemente wie z. B. Bahnhöfe, Busstationen oder Park \& Ride können einfach als Symbol/Piktogramm ausgedruckt und ausgeschnitten und so an die Lernenden verkauft werden. Das Budget sollte auf jeden Fall soweit durchdacht sein, dass sich mit dem zur Verfügung gestellten Geld nicht alles ausgehen kann, was man an Verkehrsnetz realisieren könnte. Es müssen, um die Lernziele zu erreichen, Interessenskonflikte und Knappheit entstehen, sodass innerhalb der Planungsgruppen Konflikte ausgetragen und Kompromisse geschlossen werden.

Nachdem die Planung vollzogen wurde, sollen die Schüler/innen, z. B. im Zuge eines Marktplatzes oder einfach als kurze Präsentation, ihr Verkehrskonzept vorstellen. Hier besteht schon die Möglichkeit, dass die anderen Lernenden bzw. die Lehrperson Aspekte des jeweiligen Verkehrsnetzes kritisch hinterfragen bzw. schlichtweg Fragen stellen, wenn es Unklarheiten gibt. 


\section{Reflexions- und Abschlussphase der Un- terrichtskonzeption}

Wie bereits besprochen, bildet die Nachbesprechung und Reflexion den wohl wichtigsten Teil im Sinne der Erzielung der angestrebten Kompetenzen und Lernziele. Sie soll den Planungsprozess auf einer höheren Ebene betrachten. Dafür steigen die Lernenden bewusst wieder aus ihren Rollen aus, um darüber reflektieren zu können. Die Reflexion kann anhand der folgenden Leitfragen stattfinden:

- Wie seid ihr bei der Planung vorgegangen? Wie habt ihr euch organisiert? Welche Aspekte/Bereiche waren euch wichtig und welche weniger? Bei welchen Aspekten ward ihr euch einig und bei welchen nicht?

- Wie erging es euch in den verschiedenen Rollen? Wie nah standet ihr Euren Rollen?

- Wie erging es euch im Aushandlungsprozess? Wer konnte seine persönlichen Interessen durchsetzen und warum? Wurde jemand benachteiligt und warum? Wo lagen hier die Schwierigkeiten und Chancen?

- Welche Ergebnisse eurer Arbeit würdet ihr als gelungen einschätzen und warum? Mit welchen Teilen seid ihr eher nicht zufrieden und warum?

- Welche weiteren Schwierigkeiten sind aufgetreten?

Die Lehrperson sollte im Planungsprozess so gut wie möglich die Gruppen beobachten, um auch ihre Außenperspektive auf die Gruppen- und damit politischen Prozesse geben zu können. Gerade bei jungen Schülerinnen und Schülern mag es vorkommen, dass Benachteiligungen und Fehler schöngeredet werden und so die Reflexion gehemmt werden kann. Es mag auch sinnvoll erscheinen, einige Schüler/innen nicht in die Planung zu integrieren, sondern als unabhängige Beobachter/innen einzusetzen. Für Ungeübte kann auch ein Leitfaden für die Beobachtung erstellt werden, der auf die obigen Reflexionsfragen abzielt. Damit kann in die Reflexion eine weitere Außensicht - abgesehen von jener der Lehrperson - integriert werden. Bei nur einer Lehrperson wird diese vermutlich sehr damit beschäftigt sein, die Ressourcen und Materialien zu verkaufen. Auch dieser Punkt könnte jedoch an Lernende delegiert werden.

\section{Zeitlicher Rahmen}

Zeitlich ist dieses Unterrichtskonzept recht intensiv, nicht nur in der Vorbereitung, sondern auch in der Durchführung. Es eignet sich deshalb wahrscheinlich am besten für einen halben Projekttag. Es sollten auf jeden Fall etwa vier Stunden dafür eingeplant werden, natürlich auch je nach Klasse und Altersstufe. Das Konzept ließe sich nämlich durchaus auch etwas komplexer gestalten und damit für eine Oberstufe anwenden. Gerade die Reflexion ist zeitlich sehr intensiv, sollten doch möglichst alle zu Wort kommen. Sie kann natürlich aber auch in schriftlicher Form durchgeführt werden, um so etwas Zeit zu sparen. Damit geht aber der Mehrwert verloren, dass möglichst viele von den Einzelreflexionen profitieren können.

\section{$6 \quad$ Fazit}

Was dieses Unterrichtskonzept auf jeden Fall leisten kann, ist ein Beitrag zur politischen Urteils- und Handlungskompetenz und eine Schärfung des Verständnisses für politische Prozesse. Es soll eine kleine Stufe auf dem Weg vom kollektiven Politikverständnis zu einem „selbstreflexiven Ich“ sein, das sich der Vielfalt an Ideen und Interessen bewusst ist und es auch versteht, sich in eine Vielzahl an Perspektiven hineinzuversetzen (Hellmuth 2009, 14 f.). Damit wird man auch dem bereits in den 1970er Jahren in Beutelsbach geforderten Prinzip der Kontroversität gerecht werden können, wonach alles, was in Wissenschaft, Wirtschaft und Politik kontrovers diskutiert wird, auch im Unterricht kontrovers dargestellt werden soll (Sander $\left.2005^{3}, 18\right)$. Nicht zuletzt kann damit auch der Beitrag zur Aufgabe der österreichischen Schule geleistet werden, junge Menschen zu selbständigem Urteil zu bringen und sie für anderen politische Denkweisen zu öffnen $(\$ 2$ SchOG).

\section{$7 \quad$ Literaturverzeichnis}

$\mathrm{BMBF}$ - Bundesministerium für Bildung und Forschung (2000): Lehrplan Geografie und Wirtschaftskunde Unterstufe. https:/www.bmbf.gv.at/schulen/unterricht/ lp/ahs9_784.pdf?4dzgm2 (17.04.2016)

$\mathrm{BMBF}$ - Bundesministerium für Bildung und Forschung (2015): Grundsatzerlass Politische Bildung. https:// www.bmbf.gv.at/schulen/unterricht/uek/pb_ grundsatzerlass_15683.pdf?4dzgm2 (16.04.2016).

Fassmann, H. (2006): Wie politisch ist die Geographie? Zum Verhältnis GW und PB. In: GW-Unterricht 101, 5-9.

Goll, T. (2012): Sprachhandeln: Verhandeln, Argumentieren, Überzeugen - eine vernachlässigte Kompetenz des Politikunterrichts. In: Weißeno, G. \& H. Buchstein (Hrsg.): Politisch Handeln. Modelle, Möglichkeiten, Kompetenzen. Bonn: Bundeszentrale für Politische Bildung, 193-209. 
Hellmuth, T. (2009): Das „selbstreflexive Ich“. Politische Bildung und kognitive Struktur. In: Hellmuth, T. (Hrsg.): Das „selbstreflexive Ich“. Beiträge zur Theorie und Praxis politischer Bildung. Innsbruck, Wien und Bozen: Studienverlag, 11-20.

Jank, W. \& H. Meyer (2011): Didaktische Modelle. 11. Auflage. Berlin: Cornelsen Verlag.

Krammer, R. (2008): Kompetenzen durch Politische Bildung. Ein Kompetenz-Strukturmodell. In: Kompetenzorientierte Politische Bildung, Informationen zur Politischen Bildung 29, 5-14.

Langhagen-Rohrbach, Ch. (2010): Raumordnung und Raumplanung. 2. Auflage. Darmstadt: Wissenschaftliche Buchgesellschaft.

Sander, W. (2005): Theorie der Politischen Bildung: Geschichte - didaktische Konzeptionen - aktuelle Tendenzen und Probleme. In: Sander, W. (Hrsg.): Handbuch Politische Bildung (=Reihe Politik und Bildung Band
32), 3. Auflage. Schwalbach/Ts.: Wochenschau Verlag, $13-47$.

SchOG - Schulorganisationsgesetz (1962): http:// www.bhak-dl.ac.at/cms/index.php?option =com docman\&task=doc_download\&gid=18\&Itemid = 129 (26.09.2016).

Sitte, W. (2001): Operativer GW-Unterricht. In: Sitte, W. \& H. Wohlschlägl (Hrsg.): Beiträge zur Didaktik des „Geographie- und Wirtschaftskunde“-Unterrichts. (=Materialien zur Didaktik der Geographie und Wirtschaftskunde Bd. 16), Wien.

Sutor, B. (1984): Neue Grundlegung politischer Bildung. Paderborn, München, Wien und Zürich: Schöningh.

Wiepcke, C. (2011): Planspiel. In: Reinhardt S. \& D. Richter (Hrsg.): Politik-Methodik. Handbuch für Sekundarstufe I und II. 2. Auflage. Berlin: Cornelsen Verlag, 63-67. 


\section{Arbeitsaufgabe - Verkehrsplanung}

Wir sind in der Stadt „Utopolis“. Dort wohnen etwa 20000 Menschen. Die Stadtplaner/innen haben jedoch bei der Planung der Stadt etwas Wesentliches vergessen: alle Verkehrswege. Deshalb wird erneut ein Planungsteam einberufen, das für die Stadt einen Verkehrsplan erstellen soll.

- Bildet Zweiergruppen und lest eure Rollenkarte. Sammelt verschiedene Verkehrsmittel und Möglichkeiten, sich durch die Stadt fortzubewegen.

- Erstellt nun mit der Karte einen Plan für den Verkehr in der Stadt. Welche Fortbewegungsmöglichkeiten werden gebraucht? Wie werden die Orte verbunden?

- Zeichnet die Verkehrsinfrastruktur in die Stadtkarte ein. Verwendet unterschiedliche Farben!

- Sammelt eure Ergebnisse gemeinsam mit den anderen Schülerinnen und Schülern, Touristinnen und Touristen, Radfahrerinnen und Radfahrern, Lieferantinnen und Lieferanten! Begründet eure gezeichneten Verkehrswege (Warum habt ihr euch für diese Verkehrsformen entschieden?)

- Entwerft einen gemeinsamen Verkehrsplan! Was wird benötigt? Diskutiert über Notwendigkeit von Verkehrsmitteln. Geht Kompromisse ein.

ACHTUNG: Ihr habt nur folgendes Budget zur Verfügung. Mehr kann sich die Stadt nicht leisten

\section{Budget zur Verfügung: 80 GE (Geldeinheiten)}

\section{Wege und Straßen}

1 Einheit Radweg - 1 GE

1 Einheit Schienen - 3 GE

1 Einheit Straße - 3 GE

\section{Brücken}

1 Radbrücke - 5 GE

1 Autobrücke - $10 \mathrm{GE}$

\section{Öffentlicher Verkehr}

1 Buslinie (inkl. 3 Stationen) - 10 GE

1 Straßenbahnlinie (inkl. 3 Stationen) - 10 GE

1 Bahnhof - 20 GE

1 Schiffslinie (inkl. 2 Stationen)- 20 GE

\section{Sonstiges}

1 Park \& Ride - 5 GE 


\section{Schüler/in}

Du wohnst mit deinen Eltern in einer Siedlung außerhalb der Stadt. Dir ist es wichtig, interessante Freizeiteinrichtungen und die Schule selbstständig zu erreichen. Du hast noch keinen Führerschein. Deine Eltern haben nicht immer Zeit, dich zu fahren.

$8<$

\section{Tourist/in}

Als Tourist/in willst du möglichst interessante Orte in der Stadt erreichen. Dich interessiert die Altstadt genauso wie die Natur um die Stadt herum. Auto hast du keines zur Verfügung. Du bist mit dem Flugzeug am Flughafen der Stadt gelandet.

$8<8$

\section{Radfahrer/in}

Radfahren ist deine große Leidenschaft, sowohl in als auch außerhalb der Stadt. Du findest es aber gefährlich, alle Wege auf großen Straßen zurückzulegen. Da du dich gerne in der Natur entspannst, wäre es dir auch sehr recht, wenn du schöne Orte außerhalb der Stadt erreichen könntest.

$8<8$

\section{Lieferant/in}

Du belieferst Fabriken und Gastronomie inner- und außerhalb der Stadt. Mit deinem LKW musst du immer pünktlich und verlässlich deine Ziele erreichen. Wichtig ist, dass du das Krankenhaus, das Industrieviertel und das Einkaufszentrum gut erreichen kannst. 